\title{
Lessons from altered states of consciousness
}

\author{
BL Negrao, M Viljoen \\ Department of Physiology, School of Medicine, Faculty of Health Sciences, University of Pretoria, South Africa
}

\begin{abstract}
Key research related to consciousness is that which investigates the neural systems that are deactivated or attenuated during altered states of consciousness, as well as during unconsciousness. This paper addresses various states of altered consciousness such as anaesthesia, sleep, vegetative states, seizures, post-LSD hallucinations, the minimally conscious state, locked-in syndrome, comatose states and thalamocortical dysrhythmia. Anaesthetics have been shown to act at all levels of integration in the central nervous system, with a wide range of known targets. Various conflicting theories regarding the anaesthetic state exist and a few are examined in this paper. In general, studies into altered states of consciousness emphasize the central role of prefrontal activity, the diffuse brain connectivity and the participation of the thalamocortical system/reticular formation in the maintenance of consciousness.
\end{abstract}

Key words: Unconsciousness; Anaesthesia; Coma; Thalamocortical dysrhythmia

Received: 10-10-2008

Accepted: 26-02-2009

\section{Introduction}

Much can be learnt about consciousness by identifying what becomes altered or absent during periods of

unconsciousness or altered states of consciousness. Studies into altered states of consciousness are unravelling the neural correlate(s) of unconscious states from those of the conscious state and therefore have a key significance in the quest to understand and explain consciousness. What follows are brief discussions of a number of conditions to show how they support what we know about the conscious state.

\section{Anaesthesia as an altered state of consciousness}

"With anaesthetic agents we seem to have a tool for producing and holding at will, and at little risk, different levels of consciousness - a tool that promises to be of great help in studies of mental phenomena." Henry K. Beecher, $1947^{1}$

Knowledge about the mechanisms of anaesthesia and research into consciousness have begun to converge. The hypothesis of anaesthetic action has changed from the unitary theory of Claude Bernard, which argues that all

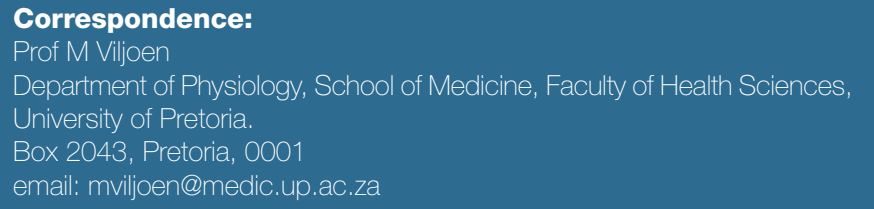

anaesthetics act at the same target in the brain, to one that selectively incorporates a multitude of neural systems and receptors. ${ }^{2}$ Anaesthetics have been shown to act at all levels of integration in the central nervous system, with a wide range of known targets. ${ }^{3}$ Targets of anaesthetics include subcellular axons, dendrites, pre- and postsynaptic membranes, as well as intracellular targets such as the neurotransmitter release systems, calcium homeostasis, buffering systems, second-messenger cascades and mitochondria. ${ }^{3}$ Cellular targets include neurons, glial cells, skeletal and cardiac myocytes, endocrine cells and cells of the immune system. ${ }^{3}$ The most common targets, however, are the neurotransmittergated ion channels of synapses, with most anaesthetics boosting the potency of inhibitory synapses, thereby causing neurons to function less vigorously and less selectively. ${ }^{4}$ Structures of the central nervous system involved in anaesthesia are the spinal cord, brainstem, cerebellum, midbrain, thalamus, reticular formation, basal nuclei, superior frontal gyrus, anterior and posterior cingulate gyrus, basal forebrain, insular cortex, prefrontal cortex, parietal and temporal association areas, occipitoparietal association cortices and occipital cortex. ${ }^{3}$ Mechanisms include anaesthetic-induced changes of proteins, hydrophobic pockets, hydrophilic crevices, protein-lipid interfaces, protein-protein interfaces, conformational states and ion channels. ${ }^{3}$

It is known that during anaesthesia the primary cortical response is diminished but not abolished and continued 
sensory cortical activation occurs. ${ }^{5}$ Information is therefore received, but said not to be perceived. Furthermore, brain metabolism during anaesthesia undergoes a decrease similar to that seen in a comatose state, with a reduction of approximately 50\% in global metabolism. ${ }^{6}$ Anaesthesia impacts on a number of mechanisms of consciousness which will be discussed in the content that follows:

\section{Anaesthesia and the information processing theory of Flohr}

The activation of N-methyl-D-aspartate (NMDA) synapses allow the formation of large-scale neuronal assemblies4, which is believed to underlie consciousness. ${ }^{7}$ The NMDA synapse could therefore serve as a principle target for anaesthetic action. ${ }^{7}$ This has indeed been shown experimentally since channel blockers, such as the drugs ketamine and phencyclidine ${ }^{4}$, working at the NMDA synapse, are known to result in a loss of consciousness. ${ }^{\text {? }}$

\section{Anaesthesia, gamma oscillations and cognitive unbinding} Cognitive binding, gamma oscillations and consciousness have been discussed in an earlier paper "Reflections on consciousness" - African Journal of Psychiatry 2009; 12 (4) Another perspective on how anaesthesia causes its effects is that the gamma oscillations of the rostral and caudal regions of the brain become uncoupled. ${ }^{8}$ It is therefore not the neural activity itself, but the integration of neural information that is disrupted. ${ }^{9}$ Gamma rhythms in the hippocampus may also be depressed, accounting for the amnesic effects associated with anaesthesia. ${ }^{8}$ Furthermore, the cerebral hemispheres also become functionally disconnected and frontal predominance arises. ${ }^{8}$ The medial orbital, dorsolateral prefrontal, frontal cortex, anterior cingulate cortex, paracentral gyrus, amygdala and basal nuclei have also all been shown to be inhibited during anaesthesia. ${ }^{8}$ These disturbances are reversed with recovery from anaesthetic agents and increased with an increase in anaesthetic depth. ${ }^{8}$ Certain anaesthetic agents are therefore believed to disrupt the large-scale functional integration of neural networks with the final common mechanism for anaesthesia assumed to be cognitive unbinding. ${ }^{9}$ This can be demonstrated experimentally by magnetic resonance imaging (MRI), which shows a definite loss of cortical connectivity under sevofluorane anaesthesia. ${ }^{9}$

\section{Quantum theory of anaesthesia}

According to Hameroff's quantum theory of consciousness, consciousness depends on the superposition of van der Waals London forces in the hydrophobic pockets of certain brain proteins. ${ }^{10}$ Therefore, according to the quantum theory of anaesthesia, anaesthetics are believed to act by binding through van der Waals London forces in the hydrophobic pockets of certain brain proteins, thereby altering the endogenous forces already present. ${ }^{10}$ This will naturally retard the electron mobility believed to be required for the quantum states necessary for consciousness. ${ }^{10}$ Anaesthetics are further believed to alter the endogenous activity by potentiating the effect of inhibitory proteins, and simultaneously inhibiting the activity of excitatory proteins in the brain. ${ }^{10}$

\section{Anaesthesia and a slight thalamic block of sensory information}

This theory suggests that hyperpolarization and therefore shunting of voltage-gated sodium and calcium channels ensures that less information is transferred to the cortex. ${ }^{5}$ This is known as the "thalamic switch" of consciousness, and is regarded as a dimmer switch as opposed to an on-off switch, since the brain is still able to respond to sensory stimuli. ${ }^{5}$ There are currently three lines of evidence that support this theory ${ }^{5}$ : Somatosensory cortical activation is depressed at low anaesthetic levels, in which there is still preserved consciousness; the anaesthetized brain can still process auditory and visual stimuli, which is naturally processed through the thalamus; and it has been shown that patients can retain the capacity to process auditory information at a degree that allows them to form implicit memories, showing that this information must pass through the thalamus en route to the cortex.

\section{Anaesthetic cascade of John and Prichep}

Depression of the brainstem by anaesthetic agents decreases the influence of the ascending reticular activating system (ARAS) on the thalamus and cortex. ${ }^{8}$ This depression of the ARAS will cause hyperpolarization of the aminobutyric acid (GABA) neurons in the nucleus reticularis. ${ }^{8}$ This in turn blocks thalamocortical reverberations and gamma-oscillations. ${ }^{8}$ Furthermore, there is an uncoupling of parietal-frontal cortical activity which interrupts cognition. ${ }^{8}$

\section{Anaesthesia and the unified narcosis theory of Alkire}

This theory simply states that the thalamocortical circuits are the targets for anaesthetic agents. Disruption of these circuits results in the loss of consciousness characteristic of anaesthesia. ${ }^{11}$

These theories emphasize the central role of the thalamocortical system, reticular formation, diffuse widespread brain activity and prefrontal activity in consciousness. MRI has shown a definite loss of cortical connectivity, and therefore neural integration, under sevofluorane anaesthesia, highlighting the importance of widespread functional integration of neural networks in consciousness. The anaesthetic cascade of John and Prichep highlights the importance of the reticular formation in consciousness, since it is believed to be the hyperpolarization of the reticular formation which inhibits the thalamocortical reverberations and gamma oscillations believed to be necessary for consciousness. Evidence supporting the theory of a slight thalamic block of sensory information as well as the unified narcosis theory of Alkire which pin-points the thalamocortical circuits as the target for anaesthetics, accentuate the significance of thalamocortical involvement in conscious experience. The anaesthetic cascade described by John and Prichep demonstrates that there is an uncoupling of parietal-frontal cortical activity, which emphasizes the essential role prefrontal neural activity plays in consciousness.

It is, however, important to note that no unitary mechanism for unconsciousness that applies to all anaesthetics exists, and different anaesthetic agents are believed to influence different neuronal elements in order to 
dampen or extinguish consciousness. ${ }^{5}$ The finding of preserved perceptual priming during unconsciousness is an important starting point from which to uncover the cognitive processes associated with consciousness. ${ }^{12}$ If this is the limit of cognitive functioning during anaesthesia, then we have a baseline from which to examine the neural correlate(s) of consciousness. ${ }^{12}$ Therefore, the illumination of the neural systems involved in this anaesthetic-induced lack of consciousness remains an important future step not only for anaesthetic researchers but also for those researchers involved in consciousness.

\section{Altered states of consciousness Sleep}

Early neuroscientists assumed that consciousness fades during sleep because the cerebral cortex shuts down completely. ${ }^{13}$ This has been invalidated by electroencephalography (EEG), which has shown that electrical activity and neural metabolism remain active during sleep. ${ }^{13}$ Deep sleep is associated with highamplitude, regular waves at a frequency of lower than 4 $\mathrm{Hz} .{ }^{14}$ These waves have synchronized pauses that interrupt the interaction between cortical regions and are therefore thought to disrupt conscious functioning. ${ }^{14}$ Waking and rapid-eye movement (REM) sleep are associated with thalamic depolarization, while thalamic hyperpolarization is present during non-REM sleep. ${ }^{15}$ During wakefulness, transcranial magnetic stimulation together with highdensity EEG induces a sustained response that spreads from the stimulated site to other cerebral areas. ${ }^{13}$ However, during non-REM sleep the neural activity elicited ceases by 150 milliseconds and remains confined to the stimulated site. ${ }^{13}$ Therefore, non-REM sleep is associated with a loss of effective cortical connectivity, resulting in a breakdown of communication between different areas of the brain. ${ }^{8}$ The activity therefore does not propagate, is rapidly dissipated and remains stereotypical regardless of the site that is stimulated. ${ }^{16}$ Criticism for the theory of a decrease in effective cortical connectivity being the cause of non-REM sleep stems from the fact that there are indeed many differences between wakefulness, REM and non-REM sleep and that the change in cortical connectivity could merely be another such difference and not the underlying cause of the fading consciousness found in sleep. ${ }^{13}$

Stimuli are unable to evoke a gamma band reset during non-REM sleep and no spontaneous gamma oscillations occur; while REM sleep is associated with spontaneous gamma activity, however, once again, there is a lack of gamma band reset, indicating that stimuli from the outside world are not perceived. ${ }^{15}$ Differences in activation of different brain areas between sleep and wakefulness do exist. The cingulate cortex decreases its activity in conditions where consciousness itself is decreased, such as deep sleep, whilst during REM sleep and wakeful attention the activity in the cingulate cortex is enhanced. ${ }^{17}$ The anterior cingulate cortex is involved in attention focusing ${ }^{17}$ and error detection ${ }^{18}$, which would explain its decreased activity during deep sleep. Furthermore, it has been shown that an attenuation of frontal association cortices occurs during REM sleep. ${ }^{19}$

During REM sleep, individuals report a conscious-like dreaming experience ${ }^{13}$, causing the coupling between wakefulness and consciousness to be disrupted. ${ }^{20}$ Tononi therefore predicts a pattern of transcranial magnetic stimulation similar to that found during wakefulness. ${ }^{13}$ Similarly, dreaming during REM sleep produces an EEG that is comparable to the one produced during waking consciousness. ${ }^{14}$ Dreaming is believed to depend on the integrity of cortical areas higher than primary cortices. ${ }^{21}$ This suggestion is supported by the maintenance of normal somatosensory and audioverbal motor imagery in hemiplegic and aphasic individuals' dreams. ${ }^{21}$ Lesions of the forebrain such as the parietotemperooccipital junction or the white matter near the orbitomesial prefrontal cortex are believed to result in the inability to dream. ${ }^{21}$ It is important to note that dreaming of a much shorter duration has been shown to occur in non-REM sleep. ${ }^{21}$ This can be explained since neurons are highly excitable during the depolarized stage of the slow oscillations associated with non-REM sleep. ${ }^{21}$ However, these stages of depolarization are interrupted about once every second by a stage of hyperpolarization, during which neural activity throughout the cerebral cortex ceases, thereby explaining the short duration of these dreams. ${ }^{21}$ Interestingly, both noradrenergic and serotonergic nuclei are silenced during REM sleep, with cholinergic nuclei being highly active. ${ }^{17}$

Emphasis is once again placed on the need for effective large-scale connectivity between different areas of the brain in order to elicit a conscious experience. During non-REM sleep transcranial magnetic stimulation remains confined to the stimulated site, highlighting the definite loss of cortical communication which may explain the dampening of consciousness found in sleep. The central role of the prefrontal cortex in consciousness is also touched upon, since REM sleep is associated with an attenuation of frontal association cortices.

In somnambulism individuals are able to use complex sensory-motor capacities. ${ }^{5}$ This, together with a desynchronized EEG, shows that these individuals are actually awake, but lack the presence of conscious prefrontal control. ${ }^{5}$ Large areas of frontal and parietal association cortices are deactivated ${ }^{22}$, gamma coherence is reduced and there is a breakdown in cortical functional connectivity. ${ }^{5}$ Using single photon emission computed tomography (SPECT) it has been shown that there is dissociation between mental and motor arousal with an increase of over $25 \%$ in blood flow to the posterior cingulate cortex and anterior cerebellum and a decrease in blood flow to the frontoparietal associative cortices, specifically the dorsolateral prefrontal cortex and left angular gyrus. ${ }^{23}$ Therefore, sleepwalking essentially involves the activation of highly selective thalamocingulate circuits with the inhibition of arousal systems. ${ }^{23}$ Variation in the specific activation pattern of the cingulate cortex results in a disparity in motor and emotional manifestations of sleepwalking. ${ }^{23}$ Deactivation of prefrontal cortices, as well as the decrease in the level of brainstem arousal ${ }^{4}$, during both sleep and somnambulism, ensure that the individual has a complete lack of insight and recall. ${ }^{23}$

Somnambulism highlights the importance of prefrontal activity in consciousness, since it has been shown that large areas of frontal and parietal association cortices are 
deactivated with a decrease in blood flow to the frontoparietal associative cortices. Furthermore, there is, once again, a breakdown in functional cortical connectivity, emphasizing yet again the importance of cerebral integration and communication between different brain areas in consciousness.

\section{Vegetative states}

Vegetative states are characterized by the absence of any sign of conscious perception and therefore vegetative patients are generally regarded as being awake, but unaware. ${ }^{6}$ Cycles of sleep and wakefulness are, however, retained, illustrated by EEG and/or the ability of the individual to open their eyes. ${ }^{17}$ In a vegetative state the brainstem is usually found to be spared, with most of the damage occurring in the cortical hemispheres. ${ }^{6}$ It is this sparing of the brainstem that is thought to maintain arousal, with metabolic dysfunction in the frontoparietal network, which contains the polymodal association cortices, possibly explaining the lack of awareness. ${ }^{6}$ The areas of the frontoparietal network whose dysfunction is associated with vegetative states are the frontal, cingulate and associative cortices, as well as the thalamus. ${ }^{24}$ Furthermore, metabolism is reduced to $40-50 \%{ }^{6}$, with the frontoparietal network of polymodal association cortices being the most impaired. ${ }^{24}$ This includes the lateral posterior parietal, prefrontal and parieto-temporal regions, as well as the midline precuneal, posterior cingulate and mesiofrontal areas. ${ }^{24}$ These areas are, interestingly, the most active areas in normal resting conscious individuals. ${ }^{24}$ Quantified fluorodeoxyglucose positron emission tomography (PET) scans in individuals that have recovered from vegetative states have elucidated the brain areas that are most impaired during a vegetative state and which return to near-normal values after recovery. ${ }^{25}$ The two central areas showing the largest post-recovery metabolic changes are the precuneus and frontoparietal association cortices. ${ }^{25}$ The precuneus has recently been shown to be involved in highly integrated functions such as visuo-spatial imagery, episodic memory retrieval and selfprocessing operations such as the first person perspective. ${ }^{26}$ Its activity has been shown to decrease during non-self referential goad-directed behaviour, emphasizing the importance of the precuneus in self-related mental representations. ${ }^{26}$ Indeed, hypometabolism of the precuneus has also been shown to occur during anaesthesia. ${ }^{26}$ It is, however, important to note that some studies have shown cerebral metabolism within the normal range in vegetative patients and, furthermore, recovery from a vegetative state is not always associated with an increase in the global metabolic rate. ${ }^{6}$

Vegetative patients who have photos of familiar faces projected onto their retina, display an activation of the occipitotemporal cortex, illustrating once again that the brain can process sensory signals in the absence of consciousness and stressing the fact that the majority of functions occur below the level of consciousness. ${ }^{20}$ Presentation of noxious somatosensory and auditory stimuli to vegetative patients results in an activation of the primary sensory cortices but a lack of activation in higher-order associative cortices. ${ }^{6}$ An altered connectivity between intralaminar thalamic nuclei and prefrontal and cingulate cortices was found by heavy water $\left(\mathrm{H}_{2}{ }^{15} \mathrm{O}\right) \mathrm{PET}^{27}$, which indicates that the residual cortical activity found in patients in a vegetative state does not allow for the level of higher-order integration considered necessary for consciousness. ${ }^{6}$ Interestingly, resumption in the connectivity between these polymodal association cortices and the intralaminar thalamic nuclei restores consciousness. ${ }^{27}$ Similarly, some vegetative states are characterized by a disconnection of long-range corticocortical and corticothalamic connections, with a partial restoration occurring during recovery. ${ }^{25}$ In some rare cases isolated cerebral networks may remain active, resulting in atypical behavioural patterns. An example of this would be the preservation of left-sided thalamocortical basal nuclei loops that allow these patients to express single words. ${ }^{6}$

Once again, the importance of prefrontal activity for the normal conscious state is elucidated since vegetative states are associated with metabolic dysfunction in the frontoparietal network. Stimuli are able to activate primary sensory cortices but, due to the impairment of the frontoparietal area, there is no activation of higher-order awareness. The central role of diffuse connectivity in consciousness is once again highlighted since disconnection of long-range corticocortical and corticothalamic connections and disconnection between polymodal association cortices and the intralaminar thalamic nuclei is found to occur in vegetative states. This simultaneously highlights the importance of the thalamocortical system in the process of consciousness.

\section{Seizures}

A seizure can be described as a hyper-synchronous neuronal discharge ${ }^{28}$, which may or may not result in a loss of consciousness. During simple partial seizures consciousness is minimally impaired, while complex partial seizures, during which automatisms may occur, are associated with the impairment of consciousness and memory. ${ }^{29}$ These automatisms are believed to be caused by electrical discharges that destroy the coalitions of the neural correlate(s) of consciousness. ${ }^{28}$ Damasio believes that automatisms are an example of a loss of extended consciousness with a preservation of the dimmest core consciousness. ${ }^{20}$

Absence seizures have been described as a state of wakeful unconsciousness ${ }^{28}$ and are associated with a loss of emotion, attention, adequate behaviour and recollection of the episode. ${ }^{20}$ Although the underlying mechanism of absence seizures is debated, thalamocortical generation has been indicated both clinically and experimentally. ${ }^{30}$ In addition, an individual undergoing an absence seizure generates abnormal oscillatory discharges in the thalamocortical circuit. ${ }^{28}$ Furthermore, widespread deactivation in the frontal and parietal association cortices has been shown by functional magnetic resonance imaging (fMRI) to occur during a seizure in which there is impaired consciousness. ${ }^{22}$ Interestingly, temporal lobe seizures, which are not associated with a loss of consciousness, do not show this widespread deactivation. ${ }^{22}$

The central role of the prefrontal network in consciousness is once again advocated, since it is believed to be the deactivation of the frontoparietal network that accounts for the impairment of consciousness found during 
seizures. The importance of the thalamocortical circuit in consciousness is moreover emphasized since absence seizures, which are associated with a loss of consciousness, are believed to be generated by abnormal thalamocortical discharges

\section{Post-LSD hallucinations}

Use of the drug Lysergic Acid Diethylamide (LSD) can result in chronic visual hallucinations in susceptible individuals. ${ }^{31}$ EEG spectral coherence has been calculated in these individuals in order to determine any possible changes in coherence found during hallucinatory experiences. ${ }^{31}$ Coherence is defined as a measure of the connectivity between brain regions and is influenced by the activity of the tracts connecting the specific areas. ${ }^{31}$ In post-LSD

hallucinations there is an increase in the regional coherence of the occipital region over many frequencies. ${ }^{31}$ This, together with a decrease in the coherence of the occipital region to other brain regions, shows that the occipital system functions in isolation. ${ }^{31}$ This internal synchronization of the occipital region, which indicates that it is less influenced by other regions, facilitates illusions and hallucinations. ${ }^{31}$ Hallucinations have indeed been described as a state of hyperattentiveness to intrinsic self-generated activity in the absence of any appropriate sensory input. ${ }^{19}$ Interestingly, hallucinations are also known to occur in blind individuals, first discovered by Charles Bonnet in 1769 and now referred to as the Charles Bonnet syndrome. ${ }^{32}$ Several factors are believed to contribute to these "release hallucinations" such as sensory deprivation ${ }^{33}$, social isolation ${ }^{33}$ and impaired central nervous system functioning ${ }^{32}$. Neural defects known to cause this condition include bilateral striate cortex atrophy ${ }^{34}$, cortical arteriovenous malformation ${ }^{35}$, suprasellar meningiomas ${ }^{32}$ and dysfunction of the visual association areas ${ }^{33}$.

The study into post-LSD hallucinations highlights the previously mentioned prominent role of diffuse cerebral connectivity in consciousness. A decrease in the connectivity of the occipital region to other brain areas results in the altered state of consciousness which facilitates hallucinations. Studies into Charles Bonnet syndrome emphasize the importance of the association areas in brain functioning, since the association areas receive and analyze information from sensory and motor cortices and thereby act as highlevel integration areas. ${ }^{36}$ Their dysregulation is associated with various altered states of consciousness.

\section{Minimally conscious state}

Patients in a minimally conscious state are unable to communicate consistently, however there is clear evidence of awareness. ${ }^{6}$ Auditory stimuli are able to activate both primary and higher-order association areas, indicating that there exists a retained capacity to integrate information due to more efficient corticocortical connectivity. ${ }^{6}$ It is important to note that this activation is dependent on the emotional importance of the applied stimulu.s. ${ }^{6}$ In a study by Di et al ${ }^{37}$, patients in a minimally conscious state presented with their own name by a familiar voice showed activation of primary auditory, as well as higher-order association temporal areas, shown by fMRI. An extraordinary case of recovery from a minimally conscious state in an individual 19 years after a traumatic brain injury has allowed researchers to pin-point the changes that occur in the transition from the minimally conscious to the fully conscious state. ${ }^{25}$ The most important findings were the changes found in the posterior midline cortices (including the cuneus and precuneus) by MRI, which were interpreted as an increase in myelinated fibre density and novel corticocortical sprouting, possibly related to axonal sprouting or neurogenesis. ${ }^{25} \mathrm{PET}$ also showed an increase in the metabolic activity of this brain region, which suggests that the posteromedial association area plays an important role in consciousness. ${ }^{25}$

The preservation of effective cortical connectivity and the ability of stimuli to activate higher-order association cortices, once again emphasizes the undeniable role of both diffuse connectivity and prefrontal activity in conscious experiences.

\section{Locked-in syndrome}

During locked-in syndrome individuals are conscious, but cannot move voluntarily due to damage to the brainstem. The precise location of the damage differs from that which produces coma in the sense that in this case it is the front of the brainstem that is damaged. ${ }^{17}$ This destroys the pathways which take the motor signals from the brain to the body, namely the corticospinal and corticobulbar pathways. ${ }^{6}$ Since the motor signals for blinking and vertical eye movements are found in the back region of the brainstem, an individual can therefore only move their eyes vertically and blink. ${ }^{17}$ This disorder, as well as research into comatose states, has enabled researchers to pin-point the arousal-producing effects of the brainstem to the back part of the brainstem.

\section{Comatose states}

Comatose states are characterized by a state of unresponsiveness in which there is a lack of arousal. ${ }^{6}$ Comatose states are associated with a lack of wakefulness, emotion, attention and purposeful behaviour. ${ }^{17}$ During a coma, only 50-70\% of normal grey-matter metabolism is found to occur, with the main decreases taking place in the thalamus, cerebellar cortex and brainstem. ${ }^{6}$ Mainly large rostral dorsomedial pontine, mesencephalic and paramedian thalamic lesions or global cortical damage have been associated with a comatose state. ${ }^{30}$ Damage to the brainstem associated with a comatose state includes the back part of the brainstem from the mid-upper pons and upward, not lower than the region where the fifth cranial nerve, the trigeminal nerve, enters the brainstem. ${ }^{17}$ The trigeminal nerve is important since it contributes the last set of information regarding the state of the organism. ${ }^{17}$ Interestingly, a section in cats $4 \mathrm{~mm}$ higher than the trigeminal nerve entry causes a major disturbance of wakefulness, whilst a section at the trigeminal nerve entry results in permanent wakefulness. ${ }^{17}$ The section $4 \mathrm{~mm}$ higher damages the acetylcholine upward projections, the downward cortical projections, as well as part of the parabrachial nucleus, whilst the section at the trigeminal nerve entry excludes the sleep-producing effects of the nucleus of the solitary tract and does not damage any higher structures. ${ }^{17}$ The region of the brainstem where damage causes loss of consciousness contains the serotonergic, cholinergic, monaminergic and parabrachial nuclei as well as the periaqueductal grey. ${ }^{17}$ Strangely, some comatose patients 
display a normal EEG, demonstrating that the function of the classical reticular formation is somehow preserved. ${ }^{17}$

However, it is important to note that some conscious patients, similarly, display an abnormal EEG. ${ }^{17}$ However, cerebral metabolism, as well as brain activation in response to sensory stimuli, can provide information regarding the presence, degree and localization of residual brain function. ${ }^{6}$

Comatose states emphasize the importance of the brainstem ARAS in the generation of consciousness. It is believed that the thalamocortical system is turned on and off by the brainstem reticular formation neuromodulators ${ }^{14}$ since they are able to modulate the cerebral cortex via projections to the intralaminar and reticular nuclei of the thalamus as well as projections to the basal forebrain nuclei and the cortex directly. ${ }^{38}$

\section{Thalamocortical dysrhythmia}

Thalmacortical dysrythmia is found in various disorders such as tinnitus, depression, Parkinson's disease and obsessive compulsive disorder, and is characterized by magnetoencephalography (MEG) as a widespread increase in the coherence of high and low frequency oscillations and an increase in theta rhythmicity. ${ }^{15}$ Furthermore, hyperpolarization of the thalamic relay or reticular thalamic nuclei cells occurs due to calcium channel deinactivation and an activation of gamma domains in the cortex due to assymetrical GABA inhibition exists. ${ }^{15}$ Low-threshold thalamic spike activity is also found in these patients during neurosurgical procedures. ${ }^{15}$

This disorder emphasizes the importance of both the thalamocortical system and gamma oscillatory activity in consciousness, since both systems are dysfunctional, resulting in this altered state of consciousness.

\section{Psychiatry and altered states of consciousness}

This paper is not written from a psychiatrist's perspective since it is considered wise to leave such writings to those intimately involved in the field of psychiatry. It is obvious that psychiatrists, perhaps more than any other profession, have the opportunity to contribute to the understanding of what consciousness entails by studying altered states of consciousness. Although much has been written about consciousness in psychiatric patients, systematic observations and short reports from those not specifically involved in formal studies of consciousness could make a significant contribution. This prospect is exciting when one starts to think in terms of not only altered states of consciousness, but also altered access to consciousness and consciousness modulated by either drugs, emotions or free will.

\section{Conclusion}

Throughout all the differing theories of the neural occurrences during altered states of consciousness, a few common concepts emerge. The thalamocortical system is an essential part of the consciousness machinery, allowing information to reach the cerebral cortex where it can be integrated to form a conscious experience. Indeed thalamocortical dysregulation has been associated with various altered states of consciousness such as anaesthesia, vegetative states, absence seizures and thalamocortical dysrhythmia. The brainstem reticular formation is also essential for consciousness since it modulates the activity of the thalamocortical system via direct projections to thalamic nuclei. Loss of consciousness, through different degrees, is associated with a breakdown in the effective connectivity of diffuse areas of the brain. Stimuli are therefore not able to spread throughout the various areas of the cortex and instead remain localized. This localization of stimulation impedes the neural integration of information by different brain regions, which is vital for consciousness. Studies into altered states of consciousness have furthermore elicited the central role of prefrontal activity in the maintenance of consciousness. Loss of consciousness is associated with the inhibition of the frontoparietal network, which contains the polymodal association cortices. This disruption ensures that there in no higher-order integration of information and, in the same way as the lack of cortical connectivity, ensures that information is not integrated to produce a conscious experience. It is, however, important to note that the acquisition, analysis and interpretation of neuroimaging data are complex and prone to errors ${ }^{6}$ and neuroimaging proof should not be regarded as absolute. However, neuroimaging techniques will undoubtedly remain an important tool that will further extend our comprehension of consciousness. In this short writing we touched on what can be learnt about consciousness from looking at altered states of consciousness. The writing is by no means a comprehensive analysis of the knowledge on consciousness that can be gained from any of the conditions mentioned. It is merely an indication, as well as a suggestion to the discipline of psychiatry to systematically observe, analyse and share their knowledge on altered states of consciousness by writing about their observations.

\section{References}

1. Beecher HK. Anesthesia's second power: probing the mind. Science 1947;105:164-6.

2. Tonner P, Scholtz J. The sinking brain: How to measure consciousness in anaesthesia. Best Pract Res Clin Anaesthesiol 2006;20(1):1-9.

3. Urban BW. Current assessment of targets and theories of anaesthesia. Br J Anaesth 2002;89(1):167-83.

4. Koch C. The quest for consciousness: A neurobiological approach. Colorado: Roberts and Company Publishers, 2004:87-104.

5. Hudetz AG. Suppressing consciousness: Mechanisms of general anesthesia. Seminars in Anesthesia, Perioperative Medicine and Pain 2006;25:196-204

6. Laureys $S$, Owen AM, Schiff ND. Brain function in coma, vegetative state, and related disorders. The Lancet Neurology 2004;3:537-46.

7. Flohr H. An information processing theory of anaesthesia. Neuropsychologia 1995;33(9):1 169-80.

8. Mashour GA. Integrating the science of consciousness and anesthesia. Anesth Analg 2006; 103(4):975-82.

9. Mashour GA. Cognitive unbinding in sleep and anesthesia. Science 2005;310:1768-9.

10. Hameroff S. Anesthesia, consciousness and hydrophobic pockets a unitary quantum hypothesis of anesthetic action. Toxicol Lett 1998:100-101:31-9.

11. Alkire MT, Haier RJ, Fallon JH. Toward a unified theory of narcosis: brain imaging evidence for a thalamocortical switch as the 
neurophysiologic basis of anesthetic-induced unconsciousness. Conscious Cogn 2000; 9:370-86.

12. Andrade J, Deeprose C. A starting point for consciousness research: reply to Thomas Schmidt. Conscious Cogn 2006;1 15:2830 .

13. Miller G. Neural communication breaks down as consciousness fades and sleep sets in. Neuroscience 2005;309:2148-9.

14. Seth AK, Baars BJ, Edelman DB. Criteria for consciousness in humans and other mammals. Conscious Cogn 2005; 14;1 19-39.

15. Sadock BJ, Sadock VA (editors). Kaplan and Sadock's comprehensive textbook of psychiatry. 8th edition. Philadelphia:Lippincott Williams \& Wilkins, 2005:575-82.

16. Massimini M, Ferrarelli F, Huber R, Esser SK, Singh H, Tononi G. Breakdown of cortical effective connectivity during sleep. Science 2005;309:2228-32.

17. Damasio A. The feeling of what happens: Body and emotion in the making of consciousness. San Diego:Harcourt Inc, 1999:234-76.

18. Miller EK, Cohen JD. An integrative theory of prefrontal cortex function. Annu Rev Neurosci 2001;24:167-202.

19. Vaitl D, Birbaumer N, Gruzelier J, Jamieson GA, Kotchoubey B, Kübler A. Psychobiology of altered states of consciousness. Psychol Bull 2005;131(1):98-127.

20. Damasio A. The feeling of what happens: Body and emotion in the making of consciousne.s. San Diego:Harcourt Inc, 1999:83-106.

21. Sadock BJ, Sadock VA (editors). Kaplan and Sadock's comprehensive textbook of psychiatry. 8th edition. Philadelphia:Lippincott Williams \& Wilkins, 2005:290-1.

22. Laureys $S$. The neural correlate of (un) awareness: lessons from the vegetative state. Trends in Cognitive Sciences 2005;9(12):556-9.

23. Bassetti C, Vella S, Donati F, Wielepp P, Weder B. SPECT during sleepwalking. Lancet North Am Ed 2000;356:484-5.

24. Laureys S, Perrin F, Schnakers C, Boly M, Majerus S. Residual cognitive function in comatose, vegetative and minimally conscious states. Curr Opin Neurol 2005; 18:726-33.

25. Laureys S, Boly M, Maquet P. Tracking the recovery of consciousness from coma. J Clin Invest 2006;1 16 (7): 1823-5.

26. Cavanna AE, Trimble MR. The precuneus: a review of its functional anatomy and behavioural correlates. Brain 2006;129(3):564-83.

27. Laureys S, Faymonville ME, Luxen A, Lamy M, Franck G, Maquet P. Restoration of thalamocortical connectivity after recovery from persistent vegetative state. Lancet North Am Ed 2000;355:1 790-1.

28. Koch C. The quest for consciousness: A neurobiological approach. Colorado: Roberts and Company Publishers, 2004:21 1-29.

29. Wikipedia [database on the Intenet]. c2001 - [cited 2008 Jan 23]. Available from: http://www.wikipedia.org

30. Schiff ND, Plum F. The role of arousal and "gating" systems in the neurology of impaired consciousness. J Clin Neurophysiol 2000;17(5):438-52.

31. Abraham HD, Duffy FH. EEG coherence in post-LSD visual hallucinations. Psychiatry Research: Neuroimaging Section 2001;107:151-63.

32. MCNamara ME, Heros RC, Boller F. Visual hallucinations in blindness: the Charles Bonnet syndrome. Int J Neurosci 1982;17(1):13-5.

33. Menon GJ, Rahman I, Menon SJ, Dutton GN. Complex visual hallucinations in the visually impaired: the Charles Bonnet Syndrome. Survey of Ophthalmology 2003;48(1):58-72.

34. Maeda K, Yasuda H, Haneda M, Kashiwagi A. Braille alexia during visual hallucination in a blind man with selective calcarine atrophy. Psychiatry Clin Neurosci 2003;57(2):227-9.

35. Alfaro A, Concepción L, Merabet L, Fernández E. An atypical presentation of visual hallucinatory experiences following prolonged blindness. Neurocase 2006;12(4):212-5.

36. Viljoen M. Stress and PNI [CD-ROM]. Pretoria: Department of Telematic Learning and Education Innovation;2004.

37. Di HB, Yu SM, Weng XC, Laureys S, Yu D, Li JQ et al. Cerebral response to patient's own name in the vegetative and minimally conscious state. Neurology 2007;68:895-9.

38. Parvizi J, Damasio A. Consciousness and the brainstem. Cognition 2001;79(1-2):135-60. 